\title{
Multi-Query Content based Image Retrieval System using Local Binary Patterns
}

\author{
Simily Joseph \\ Research Scholar \\ Department of Computer Applications \\ Cochin University of Science and Technology
}

\author{
Kannan Balakrishnan \\ Reader \\ Department of Computer Applications \\ Cochin University of Science and Technology
}

\begin{abstract}
Content Based Image Retrieval systems open new research areas in Computer Vision due to the high demand of image searching methods. CBIR is the process of finding relevant image from large collection of images using visual queries. The proposed system uses multiple image queries for finding desired images from database. The different queries are connected using logical AND operation. Local Binary Pattern (LBP) texture descriptors of the query images are extracted and those features are compared with the features of the images in the database for finding the desired images. The proposed system is used for retrieving similar human face expressions. The use of multiple queries reduces the semantic gap between low level visual features and high level user expectation. The experimental result shows that, the use of multiple queries has better retrieval performance over single image queries.
\end{abstract}

\section{General Terms}

Image Processing, Content Based Image Retrieval

\section{Keywords}

Feature Extraction, Query By Example, Texture Analysis.

\section{INTRODUCTION}

As the volume of multimedia information increases, highly intelligent and sophisticated methods are needed for better storage and retrieval. The power of digital media and the advances in Information Technology offers enormous image achieves and repositories for efficient information processing. In early days textual annotation methods were used for image storage and retrieval. Text based retrieval process exhibits a number of limitations. In those systems textual annotations are added manually, the process is subjective and incomplete. Text based search gives semantically similar images and content based search gives visually similar images [1]. Non-textual features are suited for efficient query processing. The development of a Content Based Image Retrieval system could meet the storage and retrieval needs of information processing and management by solving the problems in text based search engines [2]. It uses visual contents such as shape, texture color features, for the retrieval of similar images. In the past decades significant progress has been made in the development of CBIR systems. In various fields such as teaching, research, industry and medical diagnosis CBIR plays a major role [3]. CBIR system retrieves image based on low level features satisfying the query condition. The mapping from visual feature to perceptual feature is difficult for a machine. Therefore sometimes the semantics of the query will not be understandable for a machine [4]. Most often the semantic of the retrieved image to the query image is different. Therefore the demand of new approaches for reducing this gap is urgent.

Facial expression is being considered as an important factor for making the machine-human interaction effective. Recent advances in pattern recognition and imaging provides the opportunity of detecting human face expressions. The unexpected change in human physical condition can be detected from facial expressions [5]. Facial expression gives a clear picture of emotional status of human being. According to M.S.Bartlett et al. facial expression provides information about affective state and cognitive state such as interest, boredom, confusion, stress etc. They have developed a system for detecting the real time emotional expressions from video [6]. An important characteristic of face is that, face is viewed as a whole rather than viewing as a set of features. But the visual properties of face can be modeled in different ways such as holistic [7], analytic [8], and hybrid [9].The proposed system is used for retrieving desired face expression satisfying the given query.

\section{RELATED WORKS}

The study by R.C. Veltkamp gives a survey on the existing CBIR system [10]. Amore (Advanced Multimedia Oriented Retrieval Engine) provides the facility of selecting a category of images. Using a kind of template matching the system retrieves similar images. Blobworld developed by the computer science division, University of California limits the search space by selecting the image category. In ImageScape, the user can draw the outline of the desired image. For matching purpose edge mapping methods are used. In iPURE (Perceptual and User friendly Retrieval of Images) initially the images are segmented and then the Individual segments are compared by computing a weighted Euclidean distance. It provides the option of relevance feedback mechanism. MARS (Multimedia Analysis and Retrieval Systems) supports the use of direct queries on low level features. By using queries with Boolean operators, the retrieval accuracy is improved. SQUID (Shape Query Using Image Database) represents the counter of the image using 3 glob shape features. User selects the boundary of an image to retrieve similar image. Several CBIR systems such as IBM's QBIC (Query by Image Content), Virage2, GIFT (GNU image finding tool), IRMA (Image Retrieval for Medical 
Applications), SPIRS (Spine Pathology and Image Retrieval System) , ImageMap, ASSERT (Automatic Search and Selection Engine with Retrieval Tools), WebMIRS etc. solves many of the problems in content based image retrieval [11]

All the above said works are based on single image queries. J. Tang et al. propose a method that uses multiple images for querying [12]. This method extracts one feature from one image and another feature from the other. The extracted features are combined and used for further similarity comparison. Another approach by B. Moghaddam et.al. allows the user to select the Regions of Interest [13]. Different regions are processed parallelly and the best ones are combined to determine the final similarity. J.R.Smith et.al. propose a method for single/multiple color extraction from multiple regions within an image [14]. O.Huseyin et al. propose a method that segments the image so that the user can select different Regions of Interest [15]. The features of the regions are used for the efficient retrieval of similar images. The method proposed by C.Zhang et.al retrieves multiple objects based on color and texture features. Objects are segmented by extracting their counters. The Region of Interest is fixed from user's feedback and Multiple Instance Learning is incorporated into the query refinement process [16].

An optical flow computation algorithm proposed in [17], identifies human facial expression from rigid and non-rigid motions. I this study the principal regions are quantitatively tracked and by using spatial and temporal motion features, a mid-level representation is computed. Face expression identification using Machine Learning is explored in [18]. An embedded system for facial expression recognition is designed by Y. Sun et al. uses Hidden Markov Model for extracting facial characteristics [19]. A survey of automatic facial expression analysis is given in [20]. According to this the facial expression analysis involves three steps which are; face detection, facial expression information extraction and classification of facial expression.

A CBIR system for human face is developed in [21]. The background feature of the image is used for retrieving similar faces. No Multi Query CBIR system for face database is reported yet. The proposed system uses eight face expressions: happiness, sadness, surprise, fear, anger, contempt, neutral and disgust. The distinguishing feature of the proposed system is the use of logical operations for combining different queries. This multi query system allows the user's to express their information need, using a combination of more than one query. Using visual interface the user submits image example and views resultant images. The features of the query images are calculated and compared with the features of the stored image. Finally, images satisfying the given query conditions are retrieved.

\section{SYSTEM ARCHITECTURE}

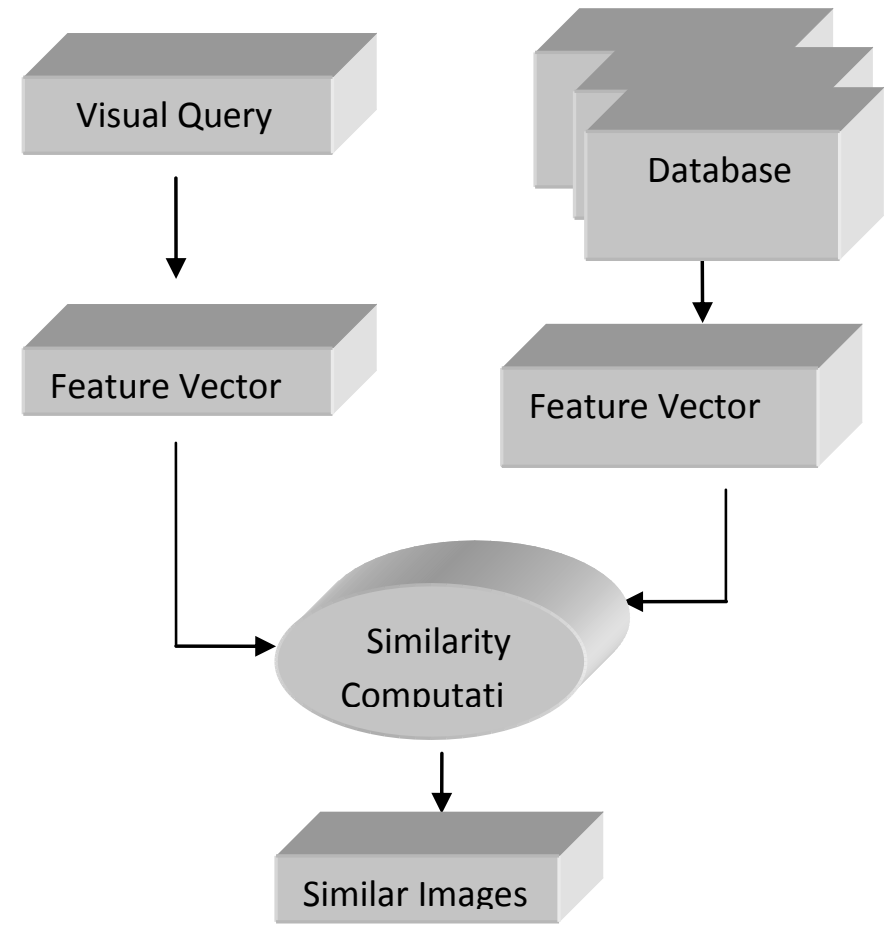

Figure 1: System Architecture

\subsection{Query By Visual Example}

Query by Visual Example retrieves images based on the low level features that are extracted from the given images. The retrieved images and the query images share some common characteristics. The multi query can be represented as $\mathrm{Q}=\mathrm{Q}_{1}, \mathrm{Q}_{2}, \mathrm{Q}_{3} \ldots \ldots \ldots \mathrm{Q}_{\mathrm{n}} . \mathrm{Q}_{1}, \mathrm{Q}_{2}$ are sub queries connected by logical operations such as AND,OR, NOT etc.

Each image contains discriminant numerical features which are extracted either globally or locally [22]. In global extraction the whole image is described and in local extraction only part of the image is described. The proposed system extracts Local binary pattern texture descriptors. Texture is the visual patterns of homogeneity. It contains information about the structural arrangements of objects and their relationships. Texture informations can be extracted for individual pixels and for a block of pixels too. The neighboring pixels in images are highly correlated. So the computation of textures of neighboring pixels yields identical results. In order to reduce the computational complexity, texture extraction methods are usually applied to a block of neighboring pixels. It is concerned with the spatial distribution of gray tones.

\subsection{Local Binary Pattern (LBP) Texture Descriptors}

LBP (Local Binary Pattern) is introduced as a gray scale invariant to obtain good classification result [23]. The local primitives such as curved edges, points, spot, flat areas etc. can be described using LBP. To generate LBP code for a 
neighborhood, the weight assigned to each pixels are multiplied with a numerical threshold. The process is repeated for a set of circular samples. As a result the local binary patterns are said to be rotation invariant. Texture over a neighborhood of pixels can be defined as the joint distribution of the gray value of a central pixel of the neighborhood say $g_{c}$ and gray value of circular pixels located at distance $P$.

$$
T=t\left(g_{c}, g_{0}, g_{1}, \cdots, g_{p-1}\right.
$$

The local texture pattern of a neighborhood can be obtained from the difference of central pixels and each pixel in the neighborhood. As the differences are independent this joint distribution can be factorized:

$$
T \approx t\left(g_{c}\right) t\left(g_{0}-g_{c}, \cdots, g_{p-1}-g_{c}\right)
$$

To make this invariant against all transformations the signs of the difference are also considered and the overall luminance $t\left(g_{c}\right)$ is ignored as it does not contribute anything to texture analysis.

$$
\begin{gathered}
T \approx t\left(s\left(g_{0}-g_{c}\right), \cdots, s\left(g_{p-1}-g_{c}\right)\right) \\
s(x)=\left\{\begin{array}{l}
1 x \geq 0 \\
0 x<0
\end{array}\right.
\end{gathered}
$$

By assigning weight, this difference is converted to a Local Binary Pattern Code which is equivalent to the local texture.

$$
L B P_{P, R}\left(x_{c}, y_{c}\right)=\sum_{p=0}^{p-1} s\left(g_{p}-g_{c}\right) 2^{P} .
$$

This equation results in the generation of $2^{P}$ LBP values.

\subsection{Similarity computation}

The query given by the user is converted to a database query. The distance function is added to the query .Then those images that satisfy the query conditions are retrieved. Image retrieval according to user's requirements is performed by measuring the similarity or dissimilarity between the features of the inputted image and the corresponding features of the image stored in database. According to the query statement the logical operations are applied and later the most similar or dissimilar images are retrieved. Depending on the logical operations in the For pages other than the first page, start at the top of the page, and continue in double-column format. The two columns on the last page should be as close to equal length as possible. query, the distance measurement varies. In each case the condition in the distance function to be satisfied depends on the logical operation.

\subsection{Query by Single Image Example}

For a user, it is easy to give a sample picture of what he is searching. The query with single image example is more compact than other type of approaches. As the system uses the actual contents of images for comparison, the retrieval performance will be high. The image contents are defined using characteristics known as features.

The similarity between the query image and the images in the database is obtained by calculating the distance of each feature of the query image and database image. In general, the distance function of query image and database image can be written as $\mathrm{D}\left(\mathrm{Q}, \mathrm{P}_{\mathrm{i}}\right)$. Where $\mathrm{Q}$ is the query image, $\mathrm{P}_{\mathrm{j}}$ is an image in the database. $\mathrm{Q}$ can be single image. The use of logical connectives helps to retrieve most significant images. Each feature has its own contribution to the distance function. The distance measure used in this study is Euclidean distance, which can be represented using the equation:

$$
\sqrt{\sum_{i=1}^{n}\left((f i(Q)-f i(P j))^{2} * w i\right)}
$$

Where $\left(f i(Q)\right.$ is the $\mathrm{i}^{\text {th }}$ feature of query image, $f i(P j)$ is the corresponding feature of the image $\mathrm{P}_{\mathrm{j}}$ in the database [24].

\subsection{Query by Multiple Image Example}

Studies have shown that queries with single images are not sufficient for better retrieval [25]. To express the information need, single image or single region is not sufficient. Existing systems with multi-image query, just combines the result obtained in the single image query processing operation. No processing of nested queries is available in present systems. The use of logical operations simplifies the retrieval process. For AND operation, the resultant image should be similar to both query images. It implies that the distance of query image to the database images $i$ and $j$ should be minimum. Therefore by taking the minimum of maximum distance between $\mathrm{Q}$ and database image will meet the query condition. The distance function is MIN (MAX (D $\left.\left(Q_{i}, P j\right)\right)$ ). Where D is the distance, $Q_{i}, P_{j}$ represent the query images and database images respectively.

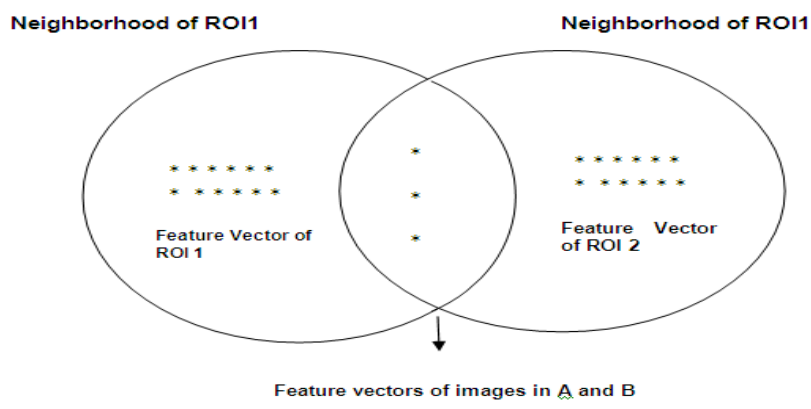

Figure 2 : Possible Feature Space for Logical AND Operation 


\section{RESULT ANALYSIS}

The efficiency of the three kinds of search is evaluated using precision. A total of 336 face expressions of eight category are selected from TFEID ( Taiwanese facial Expression Image Database) [26] . Query images are selected at random from the test database. Precision- the percentage of retrieved images that are correct, is used as the measure for result analysis.

$$
\text { Precision }=(A \cap B) / A
$$

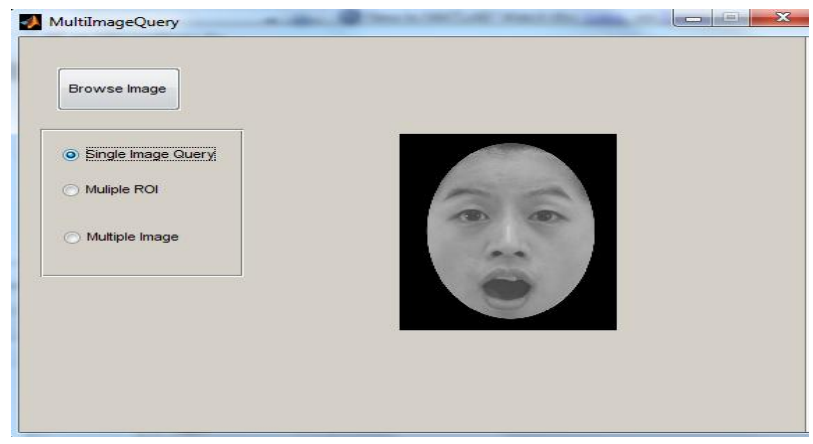

Where $\mathrm{A}$ is the number of retrieved images and $\mathrm{B}$ is the number of relevant images.

Figure 3 and Figure 4 shows the result obtained for different query images. In single image query the given face expression was surprise. In multiple image queries, two neutral expression images are given. The logical operation used was AND. The precision obtained are shown in Table 1.Multi query retrieval does help to improve system performance. As more queries are employed, higher the system performance.

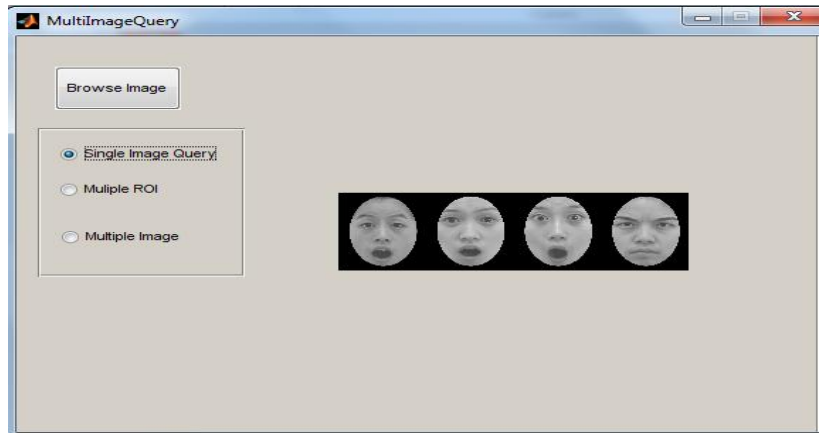

Figure 3: Result of Single Image Query
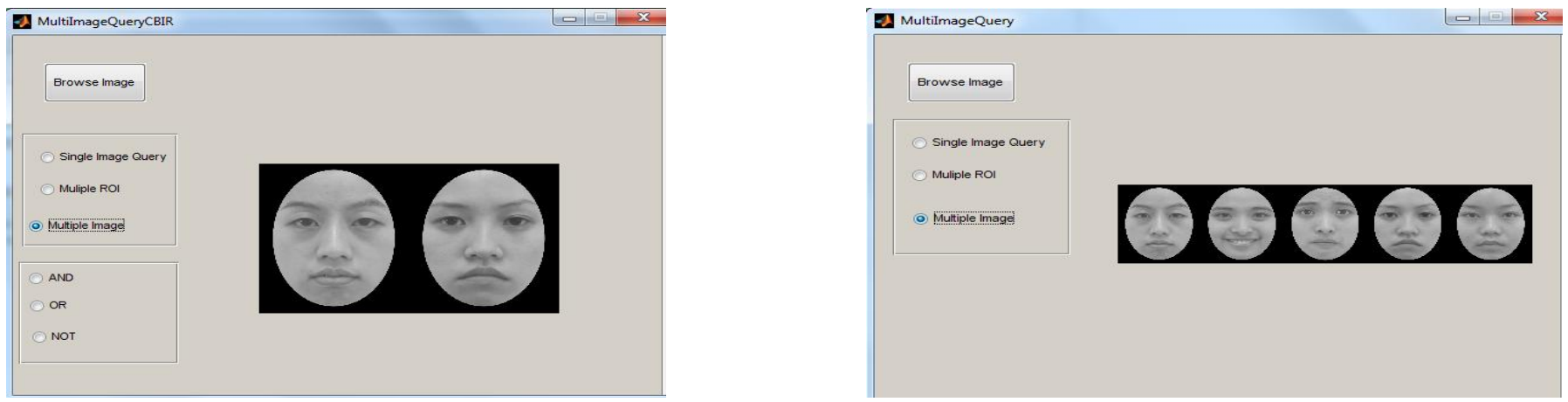

Figure 4: Result of Multiple Image Query

Table 1: Precision Obtained for Different Queries

\begin{tabular}{|l|l|l|l|l|l|}
\hline $\begin{array}{l}\text { Query } \\
\text { Type }\end{array}$ & \multicolumn{5}{|c|}{ Precision } \\
\hline & Query 1 & Query 2 & Query 3 & Query 4 & Query5 \\
\cline { 2 - 6 } $\begin{array}{l}\text { Single } \\
\text { Image }\end{array}$ & 66.67 & 69.23 & 70 & 71.43 & 75 \\
\hline
\end{tabular}

\section{CONCLUSION}

An efficient Content Based Image Retrieval system using multi query example is designed .The system is tested with human face expressions. Local Binary Pattern descriptors are used for retrieving similar images. Depending on query condition a group of images are retrieved. The use of systems that support multiimage promises better results than single image query system.

\begin{tabular}{|l|l|l|l|l|l|}
\hline $\begin{array}{l}\text { Query } \\
\text { Type }\end{array}$ & \multicolumn{5}{|c|}{ Precision } \\
\hline & Query 1 & Query 2 & Query 3 & Query 4 & Query5 \\
\cline { 2 - 6 } $\begin{array}{l}\text { Multiple } \\
\text { Image }\end{array}$ & 75 & 76.92 & 77.78 & 78.57 & 80 \\
\hline
\end{tabular}

The retrieval accuracy can be increased by query refinement by collecting user feedback.

\section{REFERENCES}

[1] H. Muller, N. Michoux, D. Bandon, and A. Geissbuhler, "A review of content-based image retrieval systems in medical applications - Clinical benefits and future 
directions," International Journal of Medical Informatics, 73(1):1-23, 2004

[2] W. C. Seng, S. H. Mirisaee, Evaluation of a contentbased retrieval system for blood cell images with automated methods , J. Med Syst, Springer 2009 ,DOI DOI 10.1007/s10916-009-9393-3.

[3] R .da. S. Torres, A.X. Falcão," Content-based image retrieval: Theory and applications", RITA, Volume XIII, Number 2, 2006, pp. 165-189.

[4] X.S. Zhou, S.Zillner, M. Moeller, M. Sintek, Semantics and CBIR: A Medical Imaging Perspective ACM 978-160558-070-8/08/07 ,CIVR'08, Canada.

[5] K. Suzuki, J. Kobayashi, T. Takeshima, and K.Yamada," Detection of unusual facial expression for human support systems", DOI. 10.1109/IECON.2008.4758509, Orlando, FL, IEEE, pp.3414-3418.

[6] M.S. Bartlett, G. Littlewort, I. Fasel and J.R. Movellan, "Real timre face detection and facial expression recognition: Development and applications to human computer interaction", Proceedings of the 2003 Conference on Computer Vision and Pattern Recognition Workshop (CVPRW'03) , U.S.A., 2003 IEEE Computer Society, pp.1-6.

[7] M. Kato, I. So, Y. Hishinuma, O. Nakamura, and T. Minami, Description and synthesis of facial expressions based on isodensity maps, Visual Computing, T. Kunii, ed., pp. 39-56. Tokyo:Springer-Verlag,1991.

[8] A.L. Yuille, D.S. Cohen, and P.W. Hallinan, Feature extraction from faces using deformable templates, ${ }^{\circ}$ Proc. Computer Vision and Pattern Recognition, pp. 104-109, 1989.

[9] K.M. Lam and H. Yan, An analytic-to-holistic approach for face recognition based on a single frontal view, IEEE Trans. Pattern Analysis and Machine Intelligence, vol. 20, no. 7, pp. 673-686, July 1998.

[10] R.C. Veltkamp, M.Tanase, "Content- based image retrieval systems: A Survey”, Technical Report UU-CS2000-34, October 2000.

[11] P. Aggarwal, H.K. Sardana, G.Jindal, "Content based medical image retrieval: Theory, Gaps and Future", ICGST-GVIP Journal, Volume 9, Issue II, April 2009, pp. 27-37

[12] J. Tang, S.Acton,An image retrieval algorithm using multiple query images, IEEE, 2003, pp. 193-196.

[13] B. Moghaddam, H. Biermann, D.Margaritis, Regionsof-interest and spatial layout for content based image retrieval, MEITC, America 2000.

[14] John R. Smith, Shih-Fu Chang, Single color extraction and image query, International Conference on Image Processing (ICIP-1995), Washington, DC

[15] C. Zhang, X. Chen, Wei-Bang Chen, An online multiple instance learning system for semantic image retrieval, Ninth IEEE International Symposium on Multimedia 2007. Taiwan.
[16] 0. Huseyin, T. Chen, H. R. Wu, Performance evaluation of multiple regions-of-interest query for accessing image databases, Proceedings of 2001 International Symposium on Intelligent Multimedia, Video and Speech Processing, Hons Konq.

[17] Y. Yacoob and L. S. Davis,” Recognizing human facial expression from long image sequences using optical flow", IEEE TRANSACTIONS ON PATTERN ANALYSIS AND MACHINE INTELLIGENCE, VOL. 18, NO. 6, JUNE 1996, pp. 636-642.

[18] J.L. Raheja, U.Kumar," Human facial expression detection from detected in captured image using back propagation neural network ", International journa of Computer science and Information Technology (IJCSIT), Vol.2, No.1, February 2010, pp.116-123.

[19] Y.Sun,Y.An, " Research on the embedded systemof facial expression Recognition Based on HMM", Proceedings of ICIME 2010, IEEE, pp. 727-731.

[20] M. Pantic and L.J.M.Rothkrantz," Automatic Analysis of Facial expresion s: The state of the art " IEEE TRANSACTIONS ON PATTERN ANALYSIS AND MACHINE INTELLIGENCE, VOL. 22, NO. 12, DECEMBER 2000, pp.1424-1445.

[21] R.Srihari, Z.Zhang and A. Rao, "Image background search: Combining object detection techniques with content-ased image retrieval(CBIR) systems", Proceedings of the IEEE workshop on Content Based Access of Image and video Libraries, 1999, U.s.A, pp.97-101.

[22] T. M. Deserno, S. Antani, L. Rodney Long, "Contentbased image retrieval for scientific literature access", Methods Inf Med 4/2009, pp. 372-380.

[23] Harwood D, Ojala T, Pietik“ainen M, Kelman S \& Davis S (1993) Texture classification by center symmetric auto-correlation, using Kullback discrimination of distributions. Technical report, Computer Vision Laboratory, Center for Automation Research, University of Maryland, College Park, Maryland. CAR-TR-678.

[24] Simily joseph, Kannan Balakrishnan, "Design of a multiimage query system for content based image retrieval", Proc of Second International conference on Multimedia and content Based Image Retrieval, ICMCBIR 2010, Banglore.

[25] S.M.M. Tahaghoghi, J.A. Thom, H.E.Williams. Are two pictures better than one? In Australian Database conference, (2001) pages 138-144, Queensland.

[26] Li-Fen Chen and Yu-Shiuan Yen. (2007). Taiwanese Facial Expression Image Database [http://bml.ym.edu.tw/download/html]. Brain Mapping Laboratory, Institute of Brain Science, National YangMing University, Taipei, Taiwan. 